\title{
Quality Assessment of Maternal and Fetal Cardiovascular Sounds Recorded From the Skin Near the Uterine Arteries During Pregnancy
}

\author{
Dagbjört H Eiríksdóttir ${ }^{1}$, Rasmus G Sæderup ${ }^{2}$, Diana Riknagel ${ }^{2,3}$, Henrik Zimmermann ${ }^{2}$, \\ Maciej Plocharski ${ }^{1}$, John Hansen ${ }^{1}$, Johannes J Struijk ${ }^{1}$, Samuel E Schmidt ${ }^{1}$ \\ ${ }^{1}$ Department of Health Science and Technology, Aalborg University, Aalborg, Denmark \\ ${ }^{2}$ Viewcare A/S, Søborg, Denmark \\ ${ }^{3}$ Department of Obstetrics and Gynaecology, Regional Hospital Viborg, Viborg, Denmark
}

\begin{abstract}
Monitoring cardiovascular activity during pregnancy is of high importance for identifying abnormal development of the fetus. Automated cardiovascular auscultation of the abdomen in both infrasonic and audible frequencies is a non-invasive method for monitoring the maternal and fetal health, including blood flow to the placenta. However, the quality of such recordings is often compromised by artifacts. The purpose of this study was to automatically identify high-quality auscultation signals. 324 recordings were obtained with two microphones placed bilaterally on the abdomen of 90 pregnant women (gestational age of 28-41 weeks), with signal duration of $30 \mathrm{~s}-180 \mathrm{~s}$. The signals were band-pass filtered to infrasonic frequencies $(2.5 \mathrm{~Hz}$ $25 \mathrm{~Hz}$ ) and audible low frequencies $(25 \mathrm{~Hz}-125 \mathrm{~Hz})$, divided into $10 \mathrm{~s}$ segments, and areas with unwanted transients were removed. Five features were calculated for segments of at least five continuous seconds. A logistic regression model was trained and tested using the identified features, obtaining a maximum classification accuracy of $92.8 \%$ for the infrasonic frequencies $(81.6 \%$ sensitivity and $97.0 \%$ specificity), and $96.1 \%$ accuracy for the audible frequencies (90.4\% sensitivity and $97.2 \%$ specificity). These results demonstrate the feasibility of automatical identification of high-quality segments at infrasonic and audible frequencies.
\end{abstract}

\section{Introduction}

Cardiovascular complications during pregnancy pose a significant threat to both fetal and maternal health. A compromise in the balance of the vascular supply between the mother and the fetus leads to adverse health effects [1]. Accurate identification and frequent monitoring of such abnormalities during pregnancy is therefore of high importance. Early intervention could reduce maternal and fetal morbidity and mortality, and facilitate clinical decision- making before the onset of irreversible consequences to the fetus [2]. Automated cardiovascular auscultation of the pregnant abdomen in both audible and infrasonic frequencies is a non-invasive procedure for obtaining information on the maternal and fetal health, including blood flow to the placenta [3-5]. However, the quality of auscultation recordings is often compromised by artifacts from interference of respiration, from sounds of the digestive system, or from unrelated ambient sounds [3]. Consequently, the placental and fetal heart sounds are often obscured, and it is therefore imperative to automatically identify and select the high-quality segments during the recording session for analysis. The aim of this study was to define and automatically identify such high-quality signals.

\section{Methods}

\subsection{Study Population}

The data in this study was obtained from a crosssectional study of women aged 18 years or older with a singleton pregnancy at 28 - 41 weeks (median of 33 weeks), conducted at Regional Hospital Viborg, Denmark, between May 1st and August 1st, 2013 [5]. A set of 324 recordings was obtained from two microphones placed bilaterally on the abdomen of 90 pregnant women, with signal duration varying from $30 \mathrm{~s}$ to $180 \mathrm{~s}$ (median of $30 \mathrm{~s}$ ), detailed in [5]. The women had body mass indices (BMI) of $26.1 \pm$ $3.3 \mathrm{~kg} / \mathrm{m}^{2}$, and maternal pulse of $80.0 \pm 10.1 \mathrm{bpm} .58$ out of the 90 pregnancies were non-pathological.

\subsection{Pre-processing}

The recordings were divided into $10 \mathrm{~s}$ non-overlapping segments (in total 3076 segments). First, the mean was subtracted to obtain zero-mean and the segments filtered with a $50 \mathrm{~Hz}$ notch filter. The signal segments were filtered with two 4th order Butterworth band-pass filters with the following cut-off frequencies: infrasonic $(2.5-25 \mathrm{~Hz})$, 
and audible $(25-125 \mathrm{~Hz})$. In the infrasonic frequencies, the heart rhythm of the mother can be detected, and in the audible spectrum sounds from the fetal heart can be heard (Fig. 1).

An envelope was generated from each segment using the Hilbert transform. The envelope was then low-pass filtered with a 4th. order Butterworth filter with a cut-off frequency of $15 \mathrm{~Hz}$. Since the segments could contain unwanted transients or interruptions of higher amplitude than the heart sounds, such as speech, abdominal sounds, or possibly friction spikes, an iterative moving average peak detector [6] was used to remove areas with these transients.

Usable segments were selected based on a criterion of a sub-segment of at least $5 \mathrm{~s}$ being present after transient removal, after which sample autocorrelation functions (ACF) of their envelopes were calculated. Shorter segments were eliminated, leaving 2457 segments at infrasonic frequency, and 2213 at audible low frequency for further analysis.

In presence of a heart rhythm, the ACF would show high repetitive peaks, and the time lag to the first highest peak after zero lag represents the duration of one cardiac cycle. If distinctive valve sounds were present in the signal, the ACF would show one or two smaller peaks in front of the first high peak. These peaks represent duration of systole and diastole (Fig. 2). To eliminate the influence of the ACF zero lag peak on the calculated features, the signal was truncated at the first local minimum and lasting $5 \mathrm{~s}$ from that point.

\subsection{Manual annotations}

The signals segments were manually annotated by visual inspection of each segment and its ACF after transient removal on a scale from 1 - 5 (Table 1). Fig. 3 shows the distribution of the quality of the segments after the manual annotation. Segments with scores 1 and 2 were labeled as Acceptable signal while the rest was labeled as Unacceptable signal. This resulted in 827 signal segments at the infrasonic frequencies and 472 at the audible low frequencies.

\subsection{Feature calculation}

Five features were computed from the signal segments: sample entropy, variance, maximum peak, relative maximum peak, all calculated from the truncated ACF, and kurtosis, calculated from the time signal segment. Sample entropy is a complexity measurement, where a lower sample entropy indicates more repetition in the signal segment. It was calculated from a downsampled $(80 \mathrm{~Hz})$ truncated autocorrelation signal with phase state $m=2$ dimensions and a tolerance $\epsilon=0.2$ times the standard deviation of the autocorrelation signal. A good quality signal was expected

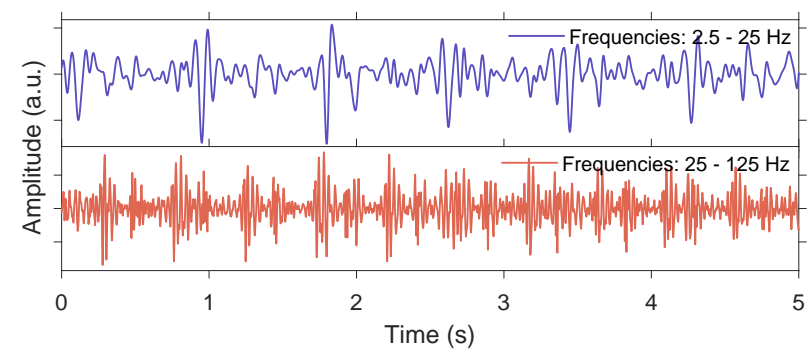

Figure 1. A 5 s sub-segment of a recording filtered at infrasonic (upper panel) and audible low (lower panel) frequencies. Pulsatile wave pattern can be seen, showing the mothers (upper panel) and fetus' (lower panel) heart rhythm.

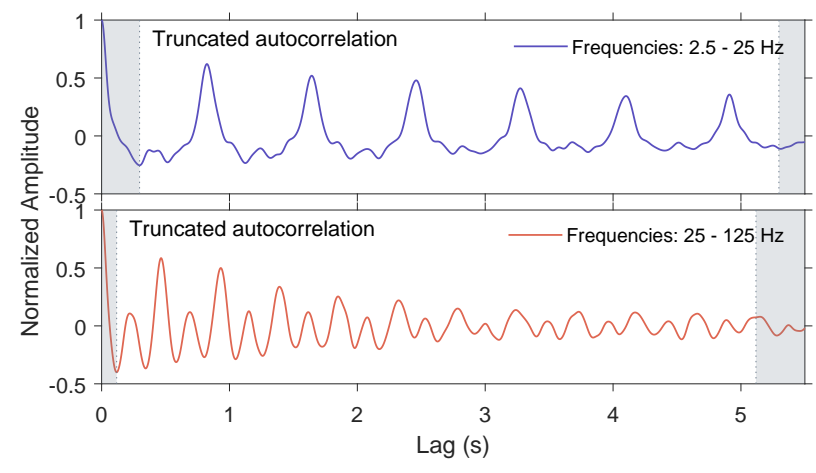

Figure 2. Autocorrelation function from an envelope of a $10 \mathrm{~s}$ signal segment filtered at the infrasonic (upper panel) and at the audible low frequencies (lower panel). Repetitive peaks can be seen.

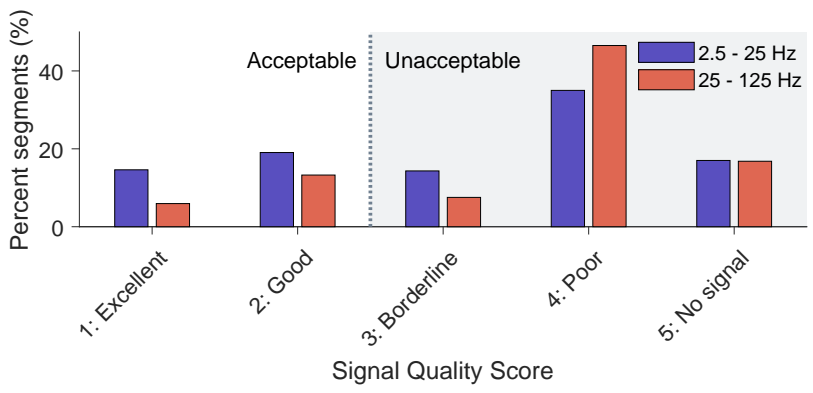

Figure 3. Quality distribution of signal segments after manual annotation. Blue bars show percentage of segments at infrasonic frequencies and red bars show percentage of segments at audible low frequencies.

to have a high peak after a lag corresponding to the interval between heart beats.

The relative maximum peak of the truncated ACF was calculated as the difference between the maximum peak and the minimum valley in the samples with a lag less than the lag of the maximum peak. This was done as visual 
Table 1. Signal quality score descriptions for manual annotations.

\begin{tabular}{|c|c|c|c|}
\hline Score & $\begin{array}{l}\text { Signal } \\
\text { Quality }\end{array}$ & $\begin{array}{l}\text { Time signal } \\
\text { description }\end{array}$ & $\begin{array}{l}\text { Autocorrelation signal } \\
\text { description }\end{array}$ \\
\hline 1 & Excellent & $\begin{array}{l}\text { Clear pulsed pattern without noise } \\
\text { (either maternal or fetal) }\end{array}$ & $\begin{array}{l}\text { High repetitive peaks present } \\
\text { (either maternal or fetal) }\end{array}$ \\
\hline 2 & Good & $\begin{array}{l}\text { Noisy pulsed pattern } \\
\text { (either maternal or fetal) }\end{array}$ & $\begin{array}{l}\text { Repetitive peaks present } \\
\text { (either maternal or fetal) }\end{array}$ \\
\hline 3 & Borderline & $\begin{array}{l}\text { Very noisy pulsed pattern } \\
\text { (either maternal or fetal) }\end{array}$ & $\begin{array}{l}\text { Low repetitive peaks } \\
\text { (either maternal or fetal) }\end{array}$ \\
\hline 4 & Poor & Noisy signal without any patterns & No repetitive peaks \\
\hline 5 & No signal & No signal or pure noise & No repetitive peaks \\
\hline
\end{tabular}

inspection showed that a false maximum peak could occur if the ACF has a gradual decrease throughout the signal. Kurtosis is a statistical measure of the tailedness of the probability function. Distributions that follow a normal distribution have a kurtosis of 3. The sound signals are expected to have peaks repeating for every heartbeat, and their distribution is thus expected to have a tailed distribution and a kurtosis larger than 3 [7].

\subsection{Post-processing}

Two thirds of the patients were randomly selected for training of the classifier while the rest was used for testing. Means and standard deviations of all features were calculated across all recordings in the training set. Both sets were then normalized by subtracting the means and dividing by the standard deviations that were calculated from the training set. This resulted in 232 recordings analyzed for training (with 1675 and 1571 processed segments for infrasonic and audible low frequencies, respectively), and 92 recordings for testing (with respectively 782 and 692 segments for infrasonic and audible low frequencies).

\subsection{Classification}

Logistic regression was used as a classification model. As the signal classification was binary, the logistic function-derived a probability equal to the conditional probability of a positive class label (Acceptable signal) and consequently the probability of a negative class label, 1 positive class (Unacceptable signal). The set of training data was used to calculate the conditional log-likelihood of the labeled classes. Two classifiers were trained and tested, one for each frequency range. Five-fold cross validation was performed on the training data to prevent overfitting. The classifier was generated using the Classification Learner application in MATLAB 2018b.

\section{Results}

Testing of the infrasonic classifier resulted in a maximum accuracy of $92.8 \%$, correctly classifying 218 out of 297 Acceptable signal segments in the testing set, corresponding to $81.6 \%$ sensitivity and $97.0 \%$ specificity. Testing of the audible classifier resulted in a maximum accuracy of $96.1 \%$, where it correctly classified 151 out of 167 Acceptable signal segments, corresponding to $90.4 \%$ sensitivity and $97.2 \%$ specificity (Table 2). Fig. 4 illustrates the ROC curves for both classifiers, with an AUC of 0.978 and 0.986 for infrasonic and audible low frequencies, respectively.

The probability of each sample belonging to the Acceptable signal class in relation to the manual annotation scores is shown in Fig. 5. Segments with quality scores 1 and 2 have higher probability for belonging to the $A c$ ceptable signal class with segments of score 1 very close to probability of 1 . Segments with quality scores 3 and 4 have a lower probability of belonging to the Acceptable signal class. Of the 92 recordings used for testing of the classifier, $49(53.3 \%)$ and $46(50.0 \%)$ had at least one Acceptable signal segment (infrasonic and audible low frequencies, respectively).

Table 2. Results of automatic quality classification for the two frequency bands.

\begin{tabular}{lllll}
\hline Frequencies & Acc. & Sens. & Spec. & AUC \\
\hline $\begin{array}{l}\text { Infrasonic } \\
(2.5-25 \mathrm{~Hz})\end{array}$ & $92.8 \%$ & $81.6 \%$ & $97.0 \%$ & 0.978 \\
$\begin{array}{l}\text { Audible } \\
(25-125 \mathrm{~Hz})\end{array}$ & $96.1 \%$ & $90.4 \%$ & $97.2 \%$ & 0.986 \\
\hline
\end{tabular}

\subsection{Discussion}

The current findings show the feasibility of automatically identifying high-quality recordings at low frequencies. Even though the number of recordings with at least 


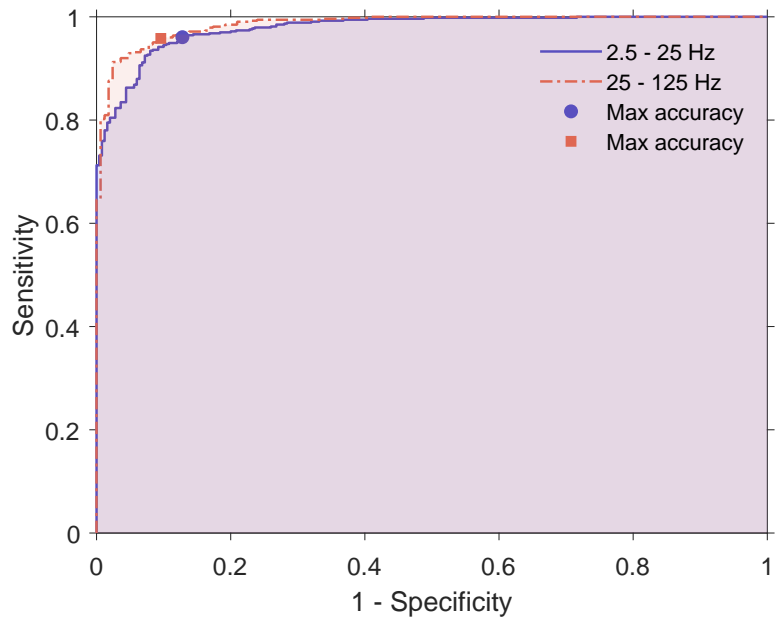

Figure 4. ROC curves for both classifiers. The blue and red marks represent the magnitude of sensitivity and specificity where the classifiers obtained maximum accuracy.

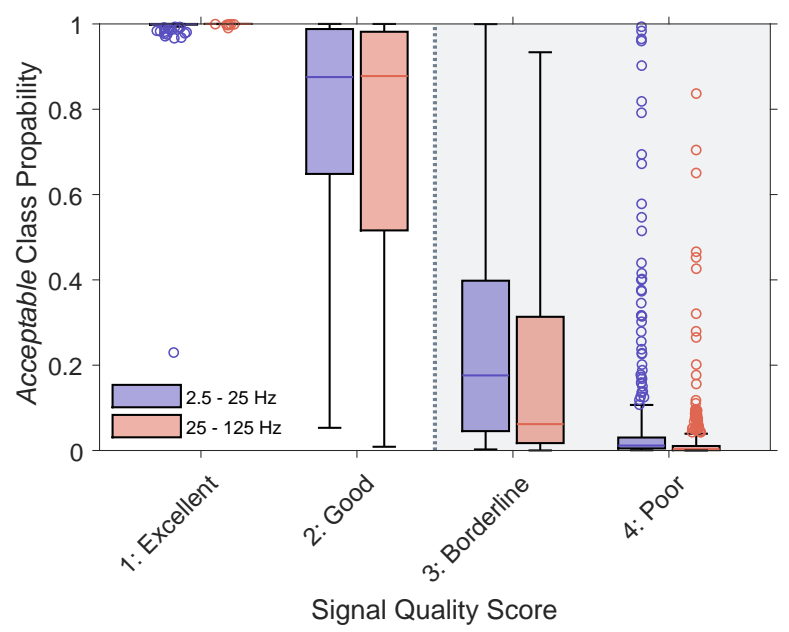

Figure 5. The probability of belonging to the Acceptable signal class for each of the manual annotation groups. Group 5 (No signal) is not shown as those signals were all eliminated before classification.

one Acceptable signal segment did not reflect the performance of the classifiers, it did show the importance of automatically identifying Acceptable from Unacceptable signal recordings, during a clinical session. Our future work could include higher frequency bands as well as improvement and expansion of the feature selection. Moreover, averaging annotations from more than one annotator would be beneficial for training the classifier, to ensure no bias in the labeling.

Investigating the class probability of the segments shows the possibility of further distinguishing the Excellent quality segments from Good segments, as they generally have a higher and more stable probability (Fig. 5). Thus, these results demonstrate the feasibility of automatically identifying high-quality sound recordings from lower quality at infrasonic and audible low frequencies.

\section{Conflict of Interest}

This project was partly funded by Viewcare A/S. RGS and $\mathrm{HZ}$ are in full employment and DR partly employed with Viewcare A/S.

\section{References}

[1] Mongraw-Chaffin ML, Cirillo PM, Cohn BA. Preeclampsia and cardiovascular disease death: prospective evidence from the child health and development studies cohort. Hypertension 2010;56(1):166-171.

[2] Imdad A, Yakoob MY, Siddiqui S, Bhutta ZA. Screening and triage of intrauterine growth restriction (IUGR) in general population and high risk pregnancies: a systematic review with a focus on reduction of IUGR related stillbirths. BMC public health 2011;11(3):S1.

[3] Varady P, Wildt L, Benyó Z, Hein A. An advanced method in fetal phonocardiography. Computer Methods and programs in Biomedicine 2003;71(3):283-296.

[4] Riknagel D, Dinesen B, Zimmermann H, Farlie R, Schmidt S, Toft E, Struijk JJ. Digital auscultation of the uterine artery: a measure of uteroplacental perfusion. Physiological measurement 2016;37(7):1163.

[5] Riknagel D, Farlie R, Hedegaard M, Humaidan P, Struijk JJ. Association between maternal vascular murmur and the small-for-gestational-age fetus with abnormal umbilical artery Doppler flow. International Journal of Gynecology Obstetrics 2017;139(2):211-216.

[6] Schmidt SE, Holst-Hansen C, Graff C, Toft E, Struijk JJ. Segmentation of heart sound recordings by a durationdependent hidden Markov model. Physiological measurement 2010;31(4):513.

[7] Springer DB, Brennan T, Ntusi N, Abdelrahman HY, Zühlke LJ, Mayosi BM, Tarassenko L, Clifford GD. Automated signal quality assessment of mobile phone-recorded heart sound signals. Journal of Medical Engineering Technology 2016; 40(7-8):342-355. PMID: 27659352.

Address for correspondence:

Dagbjört H Eiríksdóttir

Department of Health Science and Technology

Aalborg University

Fredrik Bajers Vej 7, C1-217, 9220, Aalborg, Denmark

E-mail: dhe@hst.aau.dk 